\title{
Partner notification and treatment for sexually transmitted infections among pregnant women in Gaborone, Botswana
}

\author{
Ogechukwu A Offorjebe ${ }^{1,2}$, Adriane Wynn $^{3}$, Neo Moshashane ${ }^{4}$, Dvora Joseph Davey ${ }^{5}$, \\ Kaitlin Arena ${ }^{1}$, Doreen Ramogola-Masire ${ }^{4,6}$, Ponatshego Gaolebale ${ }^{7}$, Chelsea

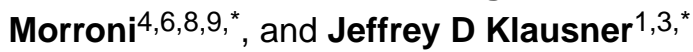 \\ ${ }^{1}$ David Geffen School of Medicine at UCLA, Los Angeles, CA, USA \\ ${ }^{2}$ Charles R. Drew University of Medicine and Science, Los Angeles, CA, USA \\ ${ }^{3}$ UCLA Fielding School of Public Health, Los Angeles, CA, USA \\ ${ }^{4}$ University of Botswana, Gaborone, Botswana \\ ${ }^{5}$ Centre for Infectious Disease Epidemiology and Research, University of Cape Town, South \\ Africa
}

${ }^{6}$ Department of Medicine, Botswana-University of Pennsylvania Partnership, Gaborone, Botswana ${ }^{7}$ Princess Marina Hospital, Gaborone, Botswana

${ }^{8}$ Wits Reproductive Health and HIV Institute (WRHI), University of the Witwatersrand, Johannesburg, South Africa

${ }^{9}$ EGA Institute for Women's Health/Institute for Global Health, University College London, London, UK

\section{Abstract}

Chlamydia trachomatis (CT), Neisseria gonorrhoeae (NG), and Trichomonas vaginalis (TV) are sexually transmitted infections (STIs) associated with adverse birth outcomes. Untreated partners contribute to high rates of STI reinfection; thus, partner notification and treatment remain important components of STI care and control. A prospective cohort study was conducted among 300 pregnant women presenting to the antenatal clinic at Princess Marina Hospital in Gaborone, Botswana who enrolled in an STI screening study. Following informed consent and sample collection for CT/NG/TV testing, participants were asked if they were willing to disclose their STI result and to deliver medications to their partner(s). Those who tested positive were asked at a follow-up appointment if they notified their partners. Among the 300 participants, 294 (98\%) said they would be willing to tell their partner(s) about their test results if they tested positive, and 284 (95\%) said they would be willing to give their partner(s) medication if the option was available. Of

Reprints and permissions: sagepub.co.uk/journalsPermissions.nav

Corresponding author: Ogechukwu A Offorjebe, David Geffen School of Medicine at UCLA, 10833 Le Conte Ave, Los Angeles, CA 90095, USA. oofforjebe@ medent.ucla.edu.

${ }^{*} \mathrm{CM}$ and JK are joint senior authors.

Declaration of conflicting interests

The authors declared no potential conflicts of interest with respect to the research, authorship, and/or publication of this article. 
those who tested positive and returned for a test of cure, 27 of $32(84 \%)$ reported that they told their partner about the results, and 20 of $32(63 \%)$ reported that their partner received treatment. Almost all pregnant women reported willingness to tell their partner the STI test result and give their partner medications. At test of cure, most women reported informing their partner, although actual treatment receipt was lower. Our findings suggest that pregnant women are willing to utilize patient-based partner notification, but actual partner treatment might be lower than intended.

\section{Keywords}

Sexually transmitted infections; Botswana; partner notification; pregnant women; sexually transmitted infection treatment

\section{Introduction}

The global prevalence of sexually transmitted infections (STIs) remains high with more than $75 \%$ of new STIs occurring in low- and middle-income countries (LMIC). ${ }^{1}$ Chlamydia trachomatis (CT), Neisseria gonorrhoeae (NG), and Trichomonas vaginalis (TV) infections are major causes of morbidity among women and are associated with adverse birth and infant outcomes. TV has been associated with numerous adverse outcomes including low birth weight and increased preterm labor. ${ }^{2-4}$ Additional studies have demonstrated an increased risk of preterm birth and spontaneous abortion among pregnant women infected with CT and NG. ${ }^{5,6}$ Untreated STIs can also be transmitted to the infant during birth, causing respiratory and eye infections. ${ }^{7}$ Further, maternal STIs may increase the risk of mother-to-child transmission of HIV. ${ }^{2,3,8}$

Untreated sex partners are a risk factor for reinfection among individuals with curable STIs, and partner notification and treatment are critical for interrupting the chain of transmission. ${ }^{9}$ Most LMICs use a patient-based partner notification strategy where index patients are given a contact slip and told to inform their partners and refer them to treatment; however, several studies in LMICs suggest that only half of partners informed via patient referral received treatment. ${ }^{10,11}$

Although there is a need to improve rates of partner notification and treatment in LMICs, particularly in sub-Saharan Africa where the STI burden remains high, ${ }^{12}$ most research on partner notification strategies has been conducted in high-income countries. ${ }^{13-16}$ In a Cochrane review of 26 randomized control trials comparing two or more partner notification strategies, only five were conducted in LMICs, ${ }^{13}$ suggesting that more research is needed to evaluate the most effective partner notification strategies in resource-limited settings.

In order to better understand the preferences and experiences of pregnant women related to partner notification and treatment for STIs, our study assessed if pregnant women were willing to disclose STI results and to deliver medications to their partner(s) in Gaborone, Botswana. We also assessed the reported partner notification and treatment results at followup. 


\section{Methods}

Our study took place in the antenatal clinic in Princess Marina Hospital in Gaborone, Botswana. Pregnant women who were 18 years old or older, $<35$ weeks gestation, and planning to return for follow-up care were eligible to participate. We offered eligible, consenting pregnant women CT, NG, and TV testing using a GeneXpert ${ }^{\circledR}$ system (Cepheid, Sunnyvale, CA) ${ }^{17}$ No incentives were provided to participants. Prior to receipt of the test results, women were asked if they would be willing to tell their partner(s) about the test result if they have an STI and provide treatment if the option was available. Those who tested positive for an STI were treated immediately, advised to inform their partner(s) and given the option of (1) bringing their partner(s) to the antenatal clinic for treatment or (2) giving their partner(s) a contact slip with instructions to receive treatment at a clinic of their choosing, per hospital standard of care. Women were not able to bring their partner medications because this was not the hospital's standard of care. Participants were advised to abstain from sex for one week following treatment. If abstaining was not possible, they were advised to use condoms to avoid reinfection. Participants were asked to return after four weeks for a test of cure using a GeneXpert ${ }^{\circledR}$ system (Cepheid, Sunnyvale, CA) and they were asked if (1) they told their partner(s) about the result and (2) the partner(s) was treated. Descriptive statistics and proportions were calculated using Stata 13 (StataCorp, College Station, TX).

The institutional review boards at the University of Botswana, the Botswana Ministry of Health, Health Research Development Committee, and Princess Marina Hospital approved the study protocol. The University of California, Los Angeles approved analyses using deidentified data.

\section{Results}

Between July 2015 and March 2016, 300 pregnant participants were enrolled in the STI screening study (Figure 1). As shown in Table 1, the median age of the study sample was 30 years and most were unmarried (76\%). The median gestational age was 27 weeks and the HIV prevalence was $22 \%$. Among the 300 participants, 294 (98\%) said they would be willing to tell their partner(s) about their test results if they tested positive, and 284 (95\%) said they would be willing to give their partner(s) medication if the option was available.

Forty-five pregnant women (15\%) tested positive for any STI, including 22 (7\%) for CT only, three (1\%) for NG only, 17 (6\%) for TV only, two (0.7\%) were dually infected with CT and NG, and one (0.3\%) was dually infected with CT and TV (Figure 2). Sixty-seven pregnant women were infected with HIV (22\%). Among those infected with HIV, 18 (27\%) were diagnosed with an STI. Among those diagnosed with an STI, 43 (96\%) were treated, $32(71 \%)$ returned for a test of cure, where $28(88 \%)$ were cured and four $(13 \%)$ retested positive, including three (9\%) for $\mathrm{CT}$ and one for $\mathrm{TV}(3 \%)$. Two participants left prior to receiving results and then were either unavailable by phone or declined to return for treatment. There was no difference in age and HIV status among those who returned for test of cure and those who did not. 
Among the 32 women who returned for a test of cure, 27 (84\%) reported that they told their partner(s) about the results, and 20 (63\%) reported that their partner(s) were treated. Of the 20 partners reported to be treated, seven (35\%) returned to the study clinic for treatment. Further among the 27 women who notified their partner(s), three (11\%) retested positive, while among the four whose partner(s) were not notified, one (20\%) retested positive.

As shown in Table 2, the median age of those who notified their partner(s) was $32(\mathrm{n}=27)$, while the median age of those who did not notify their partner(s) was $26(n=5)$. Among pregnant women living with HIV, 12 (85\%) notified their partner(s), which is similar to the notification rate among women uninfected with HIV (83\%). Among unmarried women, 24 notified their partners (83\%) and all three of the married women reported that they told their partner(s). In terms of STIs, $100 \%$ of those infected with TV notified their partner(s), while $75 \%$ and $71 \%$ of those infected with NG and CT, respectively, told their partners.

\section{Discussion}

Our study evaluated partner notification and treatment outcomes among pregnant women participating in an STI screening study in Gaborone, Botswana. We found that prior to screening, nearly all participants reported they would be willing to tell their partner about the STI results and provide medications. At follow-up, most reported that they informed their partner; however, only a little more than half reported that they knew their partner received treatment. Our findings suggest that pregnant women are willing to utilize patientbased partner notification, but actual partner treatment might be lower than intended.

Currently, there is no single optimal strategy identified for partner notification and treatment for STIs. ${ }^{13}$ Options for partner notification include provider referral (i.e. health care professional is responsible for informing partner), patient-based partner referral (i.e. patient is responsible for the partner notification), conditional referral (i.e. patient is encouraged to notify the partner, with the understanding that the provider will contact the partner if he/she does not visit the health service by a certain date), and patient-delivered partner therapy (i.e. patient takes medication to the partner). A Cochrane Review of these four strategies found that patient-delivered partner therapy was associated with decreased reinfection rates and increased numbers of partners treated compared to patient-based partner referral. ${ }^{13}$ In addition, a study of patient-delivered partner therapy for TV infection found that patientdelivered partner therapy was more cost-effective than standard partner referral. ${ }^{13}$ Some important limitations of patient-delivered partner therapy include potential adverse drug reactions and missed opportunities for counseling and testing for other STIs, including HIV. 18

In a comparison study of patient-based partner referral and patient-delivered partner therapy in South Africa, most of the women who were diagnosed with an STI chose patientdelivered partner therapy and nearly all partners reportedly took medication. ${ }^{19}$ The former findings are consistent with the high-intended willingness of participants in our study to deliver medication to their partner(s). 
Our study had several limitations. First, the study took place at a single site and has a relatively small sample size. Thus, our results may not be representative of pregnant women from other antenatal clinics in Botswana. However, it is important to note that the median age, proportion married, and HIV prevalence are consistent with the 2007 Botswana Family Health Survey IV Report and other nationally representative surveys. ${ }^{20,21}$ In addition, we were only able to obtain follow-up information from those returning for test of cure. However, a comparison between those who did and did not follow-up demonstrated no statistically significant difference in terms of age and HIV status. Furthermore, information on partner notification and treatment was based on index case reports. Our results might be influenced by social desirability bias and participants may have overreported willingness to notify their partner and provide treatment in order to present themselves favorably to the research team.

Despite the previously mentioned limitations, this study is one of the few on partner notification in sub-Saharan Africa, where the STI burden is high. Given the adverse birth and infant outcomes associated with STIs among pregnant women, identifying and implementing appropriate partner notification strategies that ensure partners are successfully notified and treated is critical. Our results suggest that more can be done to increase the number of partners that are notified and treated through strategies such as patient-delivered partner therapy. Novel approaches to partner notification and treatment should be assessed as a means to prevent reinfection in those with STIs in LMICs like Botswana. Future qualitative studies, additional antenatal clinic sites, studies with larger sample sizes, and investigation of actual uptake and management of patient-delivered partner therapy are necessary.

\section{Acknowledgments}

The authors acknowledge the UCLA Center for World Health, David Geffen School of Medicine at UCLA Short Term Training Program, Botswana-University of Pennsylvania Partnership and Antenatal Clinic, Princess Marina Hospital. They also thank Cepheid (Sunnyvale, CA) for donating the testing supplies.

\section{Funding}

The authors received no financial support for the research, authorship, and/or publication of this article.

\section{References}

1. Mayaud P, Mabey D. Approaches to the control of sexually transmitted infections in developing countries: old problems and modern challenges. Sex Transm Infect. 2004; 80:174-182. [PubMed: 15169997]

2. Sutton MY, Sternberg M, Nsuami M, et al. Trichomoniasis in pregnant human immunodeficiency virus-infected and human immunodeficiency virus-uninfected Congolese women: prevalence, risk factors, and association with low birth weight. Am J Obstet Gynecol. 1999; 181:656-662. [PubMed: 10486480]

3. McClelland RS, Sangare L, Hassan WM, et al. Infection with Trichomonas vaginalis increases the risk of HIV-1 acquisition. J Infect Dis. 2007; 195:698-702. [PubMed: 17262712]

4. Cotch MF, Pastorek JG 2nd, Nugent RP, et al. Trichomonas vaginalis associated with low birth weight and preterm delivery. The vaginal infections and prematurity study group. Sex Transm Dis. 1997; 24:353-360. [PubMed: 9243743]

5. Gencay M, Koskiniemi M, Amma“la“ P, et al. Chlamydia trachomatis seropositivity is associated both with stillbirth and preterm delivery. APMIS. 2000; 108:584-588. [PubMed: 11110046] 
6. Liu B, Roberts CL, Clarke M, et al. Chlamydia and gonorrhoea infections and the risk of adverse obstetric outcomes: a retrospective cohort study. Sex Transm Infect. 2013; 89:672-678. [PubMed: 24005255]

7. World Health Organization. Global strategy for the prevention and control of sexually transmitted infections: 2006-2015 breaking the chain of transmission. www.who.int/reproductivehealth/ publications/rtis/9789241563475/en/ (accessed 18 May 2016)

8. Adachi K, Klausner JD, Bristow CC, et al. Chlamydia and Gonorrhea in HIV-infected pregnant women and infant HIV transmission. Sex Transm Dis. 2015; 42:554-565. [PubMed: 26372927]

9. CDC. Sexually transmitted diseases treatment guidelines, 2015. MMWR. 2015; 64:10. [PubMed: 25590680]

10. Mcbride K, Goldsworthy RC, Fortenberry JD. Formative design and evaluation of patient-delivered partner therapy informational materials and packaging. Sex Transm Infect. 2009; 85:150-155. [PubMed: 19103640]

11. Alam N, Chamot E, Vermund S, et al. Partner notification for sexually transmitted infections in developing countries: a systematic review. BMC Public Health. 2010; 10:19. [PubMed: 20082718]

12. Newman L, Rowley J, Vander Hoorn S, et al. Global estimates of the prevalence and incidence of four curable sexually transmitted infections in 2012 based on systematic review and global reporting. PLoS One. 2015; 10:e0143304. [PubMed: 26646541]

13. Ferreira A, Young T, Mathews C, et al. Strategies for partner notification for sexually transmitted infections, including HIV. Cochrane Database Syst Rev. 2013(10):CD002843.

14. Oxman AD, Scott EA, Sellors JW, et al. Partner notification for sexually transmitted diseases: an overview of the evidence. Can J Public Health. 1994; 85:S41-S47. [PubMed: 7987758]

15. Macke BA, Maher JE. Partner notification in the United States: an evidence-based review. Am J Prev Med. 1999; 17:230-242. [PubMed: 10987639]

16. Trelle S, Shang A, Nartey L, et al. Improved effectiveness of partner notification for patients with sexually transmitted infections: systematic review. BMJ. 2007; 334:354. [PubMed: 17237298]

17. Wynn A, Ramogola-masire D, Gaolebale P, et al. Acceptability and feasibility of sexually transmitted infection testing and treatment among pregnant women in Gaborone, Botswana, 2015. Biomed Res Int. 2016; 2016:1251238. [PubMed: 27119076]

18. Young T, De kock A, Jones H, et al. A comparison of two methods of partner notification for sexually transmitted infections in South Africa: patient-delivered partner medication and patientbased partner referral. Int J STD AIDS. 2007; 18:338-340. [PubMed: 17524196]

19. Golden MR, Whittington WL, Handsfield HH, et al. Effect of expedited treatment of sex partners on recurrent or persistent gonorrhea or chlamydial infection. N Engl J Med. 2005; 352:676-685. [PubMed: 15716561]

20. Botswana family health survey IV report, 2007. Gaborone: Government of Botswana, Central Statistics Office, and United Nations Children' Fund; 2009.

21. Statistics Botswana NACA and Ministry of Health. Botswana AIDS Impact Survey 2013 (BAIS IV 2013). http://botswana.microdatahub.com/index.php/catalog/14 (accessed 9 January 2017) 


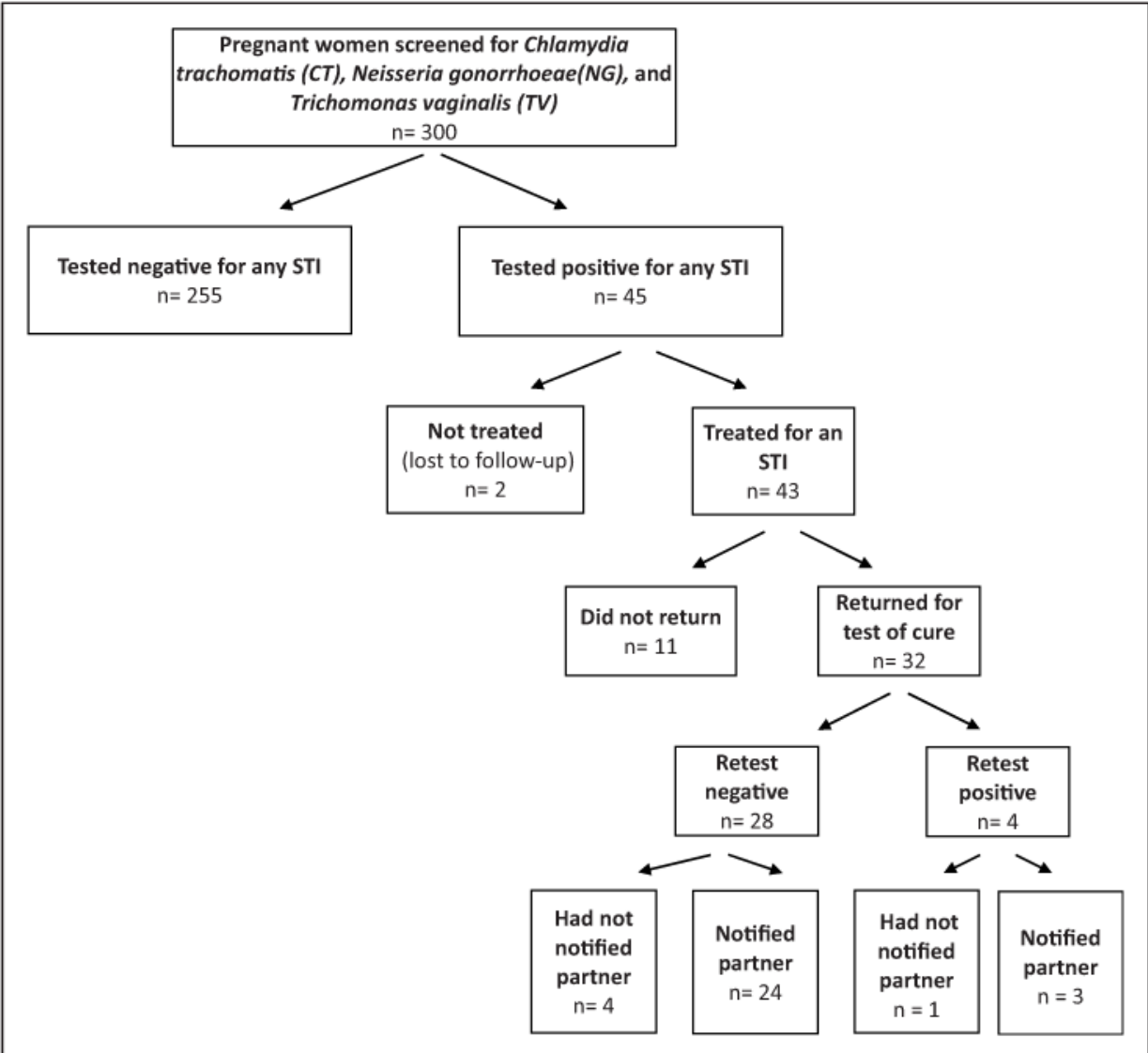

Figure 1.

Study participant flow $(\mathrm{n}=300)$. 


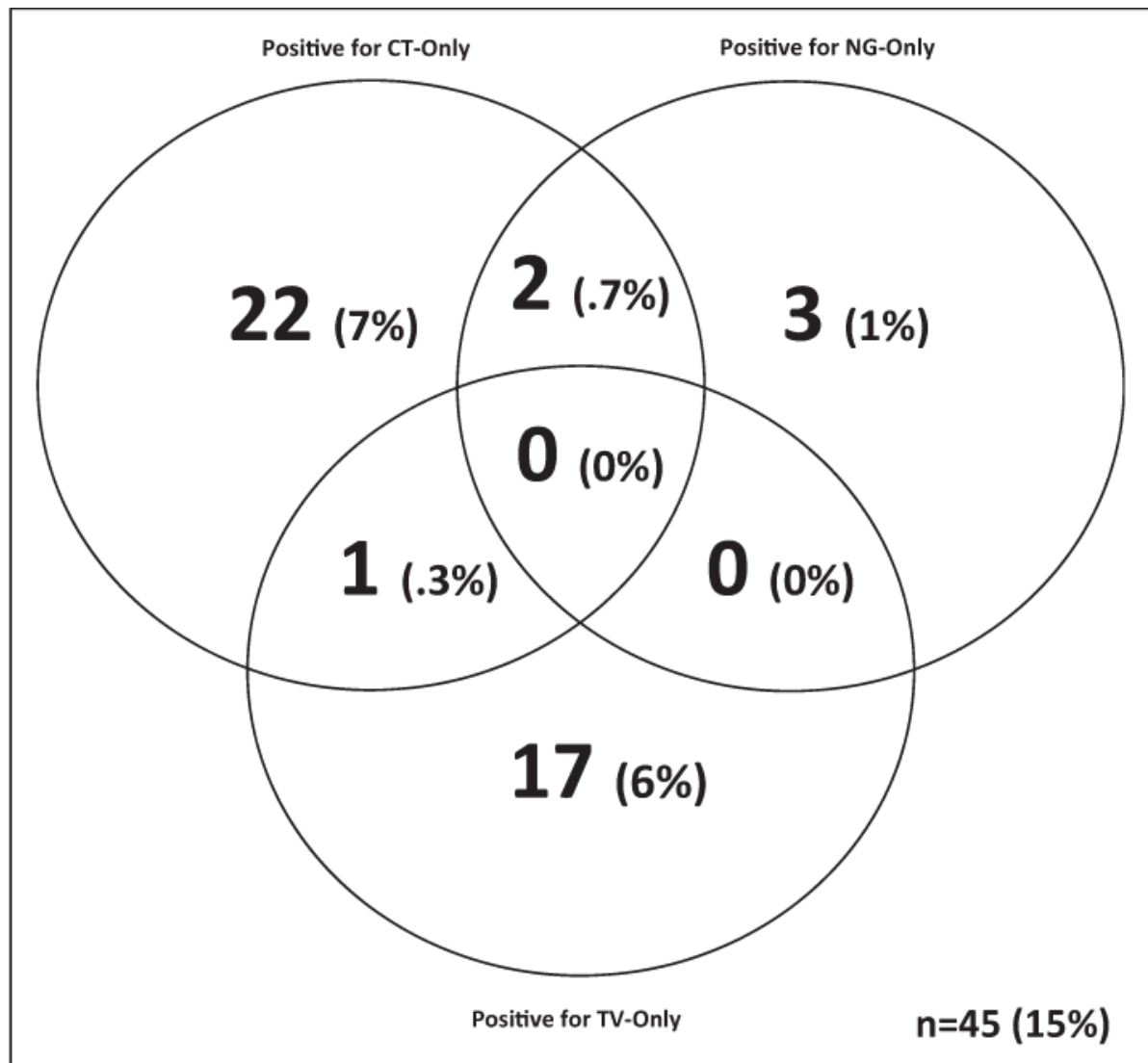

Figure 2.

Number of STIs and coinfections $(n=45)$. 


\section{Table 1}

Characteristics of study participants enrolled in the Chlamydia trachomatis, Neisseria gonorrhoeae, and Trichomonas vaginalis screening study in Gaborone, Botswana $(\mathrm{n}=300)$.

\begin{tabular}{|c|c|c|c|}
\hline & $\mathbf{n}$ & $\%$ & $95 \% \mathrm{CI}$ \\
\hline Total & 300 & 100 & \\
\hline Age in years, median (IQR) & 30 & $(26-35)$ & \\
\hline \multicolumn{4}{|l|}{ Marital status } \\
\hline Single & 228 & 76 & \\
\hline Married & 69 & 23 & \\
\hline Missing & 3 & 1 & \\
\hline \multicolumn{4}{|l|}{ Education } \\
\hline Junior secondary or less & 88 & 29 & \\
\hline Senior secondary & 89 & 30 & \\
\hline Tertiary & 119 & 40 & \\
\hline Missing & 4 & 1 & \\
\hline Gestational age weeks (LNMP), median (IQR) & 27 & $(21-32)$ & \\
\hline \multicolumn{4}{|l|}{ HIV status } \\
\hline Positive & 67 & 22 & \\
\hline Negative & 229 & 76 & \\
\hline Unknown & 4 & 1 & \\
\hline Positive for an STI & 45 & 15 & $0.11-0.20$ \\
\hline Chlamydia trachomatis (any) & 25 & 8 & $0.05-0.11$ \\
\hline Neisseria gonorrhoeae (any) & 5 & 1.7 & $0.005-0.04$ \\
\hline Trichomonas vaginalis (any) & 18 & 6 & $0.04-0.09$ \\
\hline Dual STI infection & 3 & 1 & \\
\hline Willing to notify partner of STI results & 294 & 98 & \\
\hline Willing to give medication to partner & 284 & 95 & \\
\hline
\end{tabular}

CI: confidence interval; IQR: interquartile range; LNMP: last normal menstrual period; STI: sexually transmitted infection. Percentages may not add up to 100 due to rounding. 


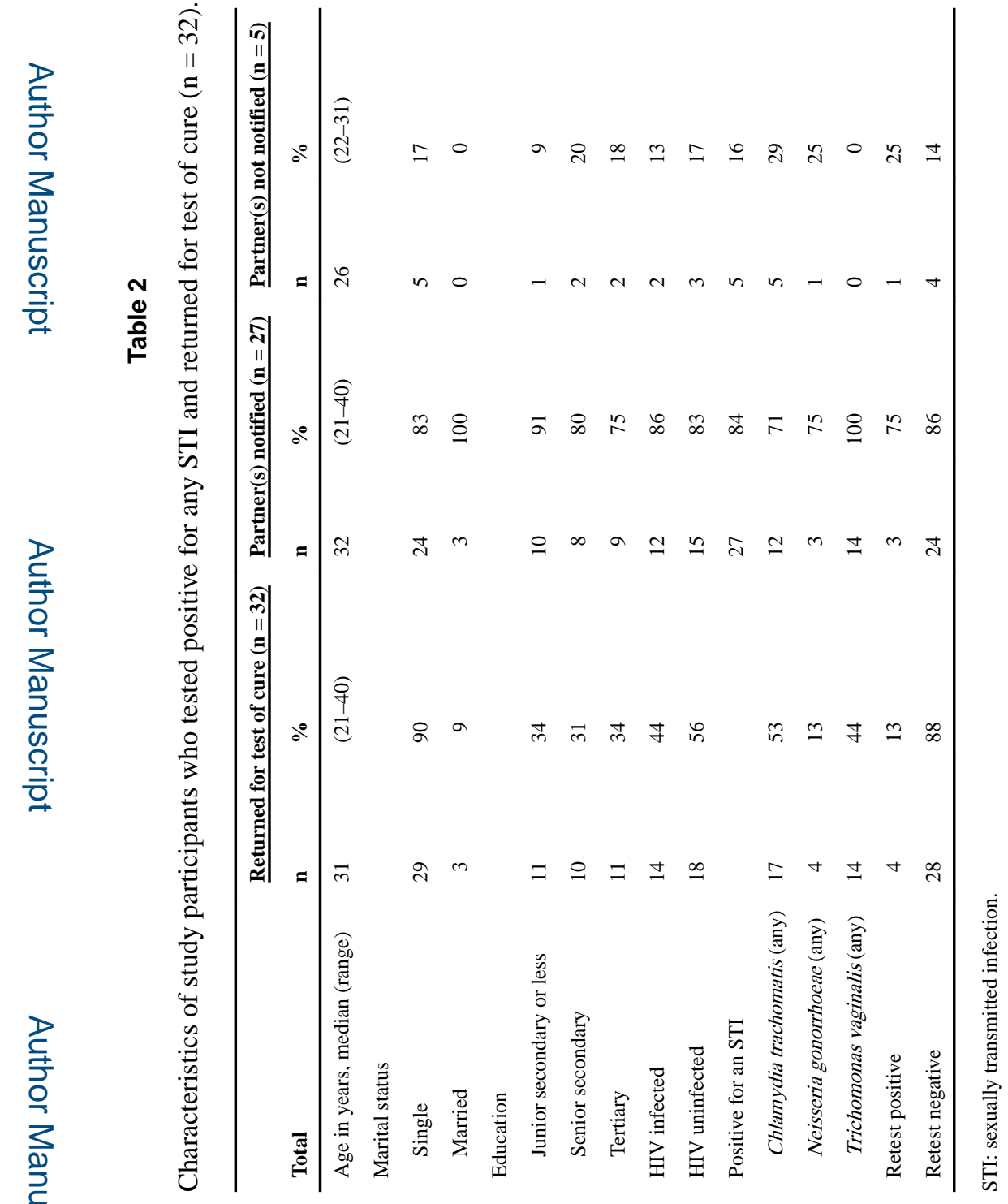

